\title{
New Perspectives in Autism Spectrum Disorder associated with Tuberous Sclerosis
}

\author{
Arianna Benvenuto ${ }^{1}$ Martina Siracusano ${ }^{1}$ Federica Graziola ${ }^{1}$ Romina Moavero ${ }^{1,2}$ Luigi Mazzone ${ }^{1,2}$ \\ Leonardo Emberti Gialloreti ${ }^{3,4}$ Paolo Curatolo ${ }^{1}$
}

${ }^{1}$ Child Neurology and Psychiatry Unit, Department of System Medicine, University of Rome Tor Vergata, Roma, Italy

2 Department of Neuroscience and Neurorehabilitation, Bambino, Gesù Children's Hospital, IRCCS, Rome, Italy

${ }^{3}$ Department of Biomedicine and Prevention, University of Rome, University of Rome Tor Vergata, Rome, Italy

${ }^{4}$ Centre for Communication and Neurorehabilitation

Research-CNAPP, Rome, Italy

J Pediatr Neurol 2017;15:123-128.

\author{
Address for correspondence Martina Siracusano, MD, Child \\ Neurology and Psychiatry Unit, Department of System Medicine, \\ University of Rome Tor Vergata, Viale Oxford 81, 00133, Rome, Italy \\ (e-mail: siracusanomartina@hotmail.it).
}

\begin{abstract}
Keywords

- tuberous sclerosis complex

- epilepsy

- autism spectrum disorder

- early intervention

- mTOR inhibitors

- parent-mediated intervention

- predictors

- risk factors

Recent advances in molecular genetics and preclinical studies of tuberous sclerosis complex (TSC) have helped to better understand the pathophysiology of TSC-related autism spectrum disorder (ASD). Furthermore, developmental studies have shown that infants with TSC begin to diverge from the neurotypical trajectories at the age of 6 months. Early abnormalities are often characterized by a delay in nonverbal cognitive skills, such as fine motor and visual reception domains followed by qualitative impairment of social communication. The expanding possibilities of an early diagnosis of TSC should increasingly allow the prompt identification of a population of infants at high risk for developing ASD. A presymptomatic diagnosis of TSC could facilitate not only the prospective investigation of developmental trajectories and early markers of ASD but also the evaluation of the efficacy of early interventions. Early identification of infants at high-risk for ASD, such as TSC infants, can allow designing individualized treatment strategies to address deficits in specific developmental domains associated with autism. The involvement of mammalian target of rapamycin (mTOR) in determining the behavioral phenotypes associated with TSC led to the hypothesis that mTOR inhibitors could also have a benefit on ASD symptoms. After the promising results from preclinical studies administrating rapamycin, clinical trials studying mTOR inhibitors are now undergoing.
\end{abstract}

\section{Introduction}

Tuberous sclerosis complex (TSC) is a genetic condition caused by a mutation in the TSC1 or TSC2 genes, ${ }^{1,2}$ coding, respectively, for hamartin and tuberin proteins, which form together a protein complex that inhibits a downstream signaling pathway, the mammalian target of rapamycin (mTOR). ${ }^{3}$ TSC is clinically characterized by multiorgan formations of hamartomatous lesions in the central nervous system (CNS), in the kidneys, liver, heart, skin, eyes, and lungs. CNS usually presents with cortical and subcortical tubers, subependymal nodules, and giant cell astrocytomas. Epilepsy is the most frequent neurological manifestation, affecting up to $85 \%$ of the patients. ${ }^{4}$ In $66 \%$ of the children with TSC, seizures onset is within the first year of life. ${ }^{5}$ Patients frequently show behavioral and psychiatric difficulties that can deeply affect their quality of life, ${ }^{4}$ like developmental delay, learning disabilities, ASD, or attention-deficit hyperactivity disorder. received

December 20, 2016 accepted after revision

February 12, 2017

published online

April 4, 2017
Issue Theme Advances in Autism Spectrum Disorder; Guest Editor: Paolo Curatolo, MD
DOI https://doi.org/ 10.1055/s-0037-1601445. ISSN 1304-2580.
Copyright $\odot 2017$ by Georg Thieme Verlag KG, Stuttgart · New York 
According to the latest Diagnostic and Statistical Manual of Mental Disorders, 5th edition criteria, autism spectrum disorder (ASD) is a neurodevelopmental condition characterized by early-onset difficulties in social communication and unusually restricted and repetitive behavior and interests. ${ }^{6}$ Prevalence of ASD is estimated to be up to 1 to $2 \%^{7,8}$ It is a long time that autistic traits in TSC have been recognized. They were already described in 1932 as impaired social contact, repetitive and stereotyped behavior, absent or abnormal speech and social withdrawal..$^{9}$ According to various authors, as well as to the criteria used for the diagnosis, ASD is commonly reckoned to be present in 17 to $63 \%$ of patients with TSC. ${ }^{10} \mathrm{~A}$ more recent study on a cohort of approximately 100 patients, evaluated by a single clinician, found a prevalence of $40 \%{ }^{11}$

The expanding possibilities of an early diagnosis of TSC should increasingly allow the prompt identification of a population of infants at high risk for developing ASD and/or epilepsy. That is why clinicians should pay further attention to the early recognition of preclinical signs, which might suggest a possible deviation from the normal developmental trajectory.

\section{Pathophysiology of Autism Spectrum Disorder in Tuberous Sclerosis}

Significant advances during the last decades in molecular biology, molecular genetics, and animal model studies of TSC have helped to understand the pathophysiology of TSC-related ASD better. It has been shown that mTOR overactivation, caused by a mutation in one of the two TSC genes, leads to global disturbances of the brain architecture and connectivity, as well as to dysregulation of the excitatory and inhibitory neurotransmission. These abnormalities lead to neural hyperexcitability, which could explain both seizures and ASD. Several animal models of TSC suggest that the neurocognitive and neurodevelopmental manifestations of TSC, including ASD, might be a direct result of cell signaling abnormalities. ${ }^{12-14}$ Indeed, mutated $\mathrm{TSC}^{+/-}$mice not only showed deficits in learning and memory but also in social behavior. ${ }^{12}$ Even if these animal models did not develop any of the brain lesions typical of TSC, they nevertheless exhibited a hippocampal dysfunction with spatial learning deficit and context-depending memory impairment. Furthermore, although autistic traits are not easily tested in mice, mutant animals failed in the common social, behavioral tests, such as the social interaction assessment and the nest building. ${ }^{12}$ These observations, as well as the lack of structural brain lesions, indicate that genetic mutations, and the subsequent cascade of events, might be directly responsible for the autistic phenotype observed in TSC. Indeed, mTOR signaling overactivity, both through direct and indirect pathways, confers a high susceptibility to ASD. It is-at least in part-responsible for the imbalance between excitation and inhibition, as it plays a role in the disruption of GABAergic interneurons during the early critical stages of neurodevelopment. ${ }^{3}$ TSC1/TSC2 dysfunction can also impair the developmental regulation of ionotropic glutamate receptors, thus enhancing glutamatergic function. ${ }^{15}$ The mTOR pathway is also associated with abnormal synaptic homeostasis and synaptogenesis, which is a well-known risk factor for ASD. ${ }^{3,16}$

\section{Epilepsy Pathways to Autism Spectrum Disorder}

In TSC children with autistic features, epilepsy often precedes the onset of ASD, raising the issue of the effects of the seizures themselves on the developmental brain. Epilepsy is considered a potential risk factor for ASD, especially when infantile spasms are present. Indeed, it has been demonstrated that although neuropsychiatric comorbidities in TSC are largely multifactorial, the only independent risk factor is seizure onset at an early age. ${ }^{17}$ It has also been hypothesized that a delayed detection and treatment of clinical seizures might increase the risk of developing ASD in patients with TSC. It seems that a shorter gap between seizure onset and antiepileptic treatment is associated with better long-term outcomes, both in terms of neurodevelopmental deficits, including lower rates of learning disabilities and ASD, and lower seizure frequency. ${ }^{18,19}$ It is reckoned that early-onset, persistent seizures, and region-specific epileptiform electroencephalogram (EEG) activity might prevent the development of an appropriate neural connectivity, leading to autistic-like behaviors and language regression.

The comorbidity of epilepsy and ASD should be seen as the last step of a common pathway starting with the genetic mutation and leading to mTOR overactivation already during fetal life. ${ }^{20}$ This activates a cascade of events, such as the imbalance between GABAergic and glutamatergic transmission, and abnormalities of white matter connectivity, which increase the susceptibility to both epilepsy and ASD. ${ }^{3}$ Since seizures may act as an epigenetic factor that worsens the final mental outcome, every effort should be made to stop the seizures as soon as possible.

Vigabatrin, an irreversible inhibitor of GABA transaminase, which also has a partial mTOR inhibition action, is considered the first-line drug for early-onset seizures in TSC. $^{21,22}$ However, seizures are preceded by a latent period of epileptogenesis; therefore, EEG biomarkers can be successfully used to detect high-risk groups by seeking and identifying epileptiform EEG abnormalities. ${ }^{23}$ A preventative treatment before the onset of clinical seizures, but after the appearance of epileptiform EEG abnormalities, has also been proposed, ${ }^{24}$ and it is the object of an ongoing multicenter study (The EPISTOP project, NCT02098759, available at: www.clinicaltrials.gov) aiming at identifying the best timing to initiate antiepileptic treatment.

\section{Early Predictors of Autism Spectrum Disorder and Neurodevelopmental Disorders}

\section{Genetic and Neuroimaging Biomarkers}

Potential biomarkers for the onset of ASD also include genetic features and cortical tubers. TSC2 mutations cause more severe histological abnormalities and more severe neurological phenotypes than mutations on the TSC1 gene. ${ }^{25}$ Recent studies investigating genotype-phenotype correlations suggested that some specific genetic mutations might confer a higher risk to develop ASD. Patients with TSC 
and ASD compared with patients with TSC but without ASD were significantly more likely to present a mutation in the TSC2 gene. ${ }^{11}$ In particular, mutations in the hamartin interaction domain of the TSC2 gene have been found to be specifically associated with ASD.

Early neuroimaging studies suggested that the presence of cortical tubers localized in the temporal lobes could be associated with a higher risk of ASD in TSC. ${ }^{26,27}$ Subsequent research showed that subjects with TSC2 mutations presented with a larger mean tuber number than subjects with TSC1 mutations. ${ }^{28}$ However, differentiating TSC patients with or without ASD on the basis of structural lesions, both in terms of localization and total number, seems to be extremely difficult. Recent data suggest that the "tuber-brain proportion" (the proportion of brain occupied by tubers) rather than their number, can be considered a potential risk factor for ASD and cognitive impairment. ${ }^{17}$ The characteristics of the tubers have been considered as well: cyst-like tubers seem to be more likely present in children with ASD than without ASD. ${ }^{11}$ In the past decade, the application of new neuroimaging techniques, including diffusion tensor imaging, helped in identifying white matter abnormalities that are more frequent in patients with ASD than in those without. ${ }^{29,30}$

\section{Clinical Signs}

The development of infants with TSC begins to diverge from the neurotypical trajectories at the age of 6 months, while a generalized developmental impairment is usually present by the age of 9 months. Abnormalities are often characterized by a delay in nonverbal cognitive skills, such as fine motor and visual reception domains (-Fig. 1). ${ }^{31}$ Early signs of atypical social communication, and in particular of visual behaviors, can also be already identified by the age of 6 months. ${ }^{31}$ The early visual perception impairment places these infants at higher risk of deficits in social function, such as visual tracking, disengagement of attention, and anticipatory responses. ${ }^{31}$ These signs seem to be more evident in the TSC population, not being associated with other "red flags" that typically identify idiopathic forms of ASD, such as social babbling or orienting to name.

The developmental trajectories of TSC infants are also influenced by the presence of ASD traits, with more severe autistic symptoms associated with more marked impairments of the developmental profile. Children with TSC and ASD had lower scores in all developmental quotients at 24 and 36 months of age, when compared with TSC children without autism or ASD. Moreover, ASD patients did not

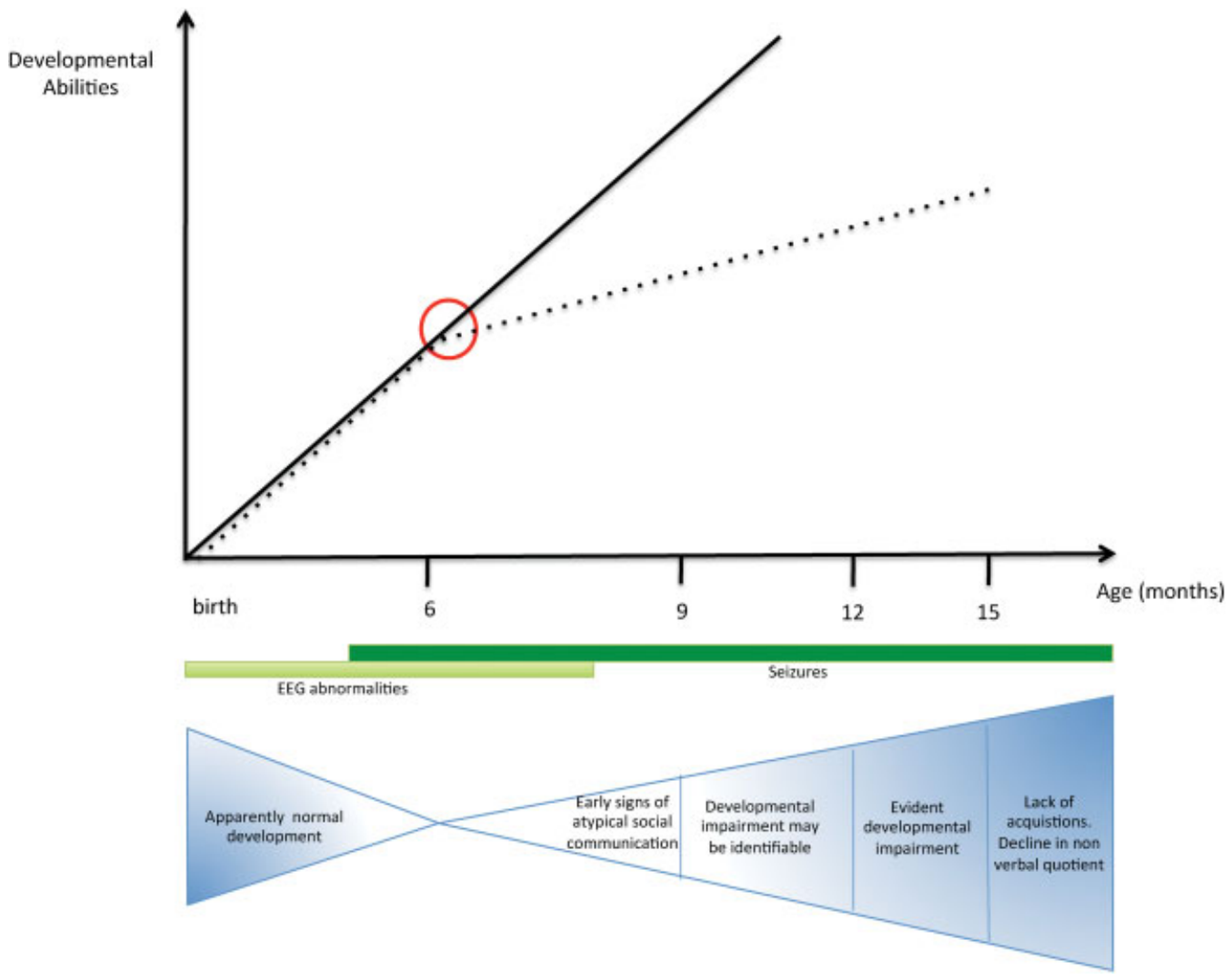

Fig. 1 Developmental and behavioral trajectories in TSC infants: TSC infants trajectories of nonverbal cognitive skills, including fine motor and visual reception domains (dotted black line), diverge from the homogeneous developmental trajectory of verbal and nonverbal abilities in "typical" children (straight black line). At 6 months early signs of atypical social communication (e.g., visual tracking, disengagement of attention, anticipatory responses) tend to be evident. This is the best time to initiate parent-mediated intervention. Evident developmental impairment (e.g., alteration of eye contact, orienting to name and motor control) is seen from 12 months on. The presence of seizures (occurring in the very first months of life in two-third of cases) worsens the developmental and behavioral trajectory. Seizures onset is preceded by a latent period of epileptogenesis during which epileptiform EEG abnormalities appear and become progressively more frequent and multifocal. TSC, tuberous sclerosis complex. 
present any change in full developmental quotients in the second and third year of life, with a significant decline in nonverbal quotients between 12 and 36 months of age. On the other hand, the non-ASD group showed significant developmental gains in nonverbal quotients, but not in

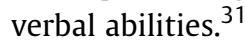

Qualitative impairment of social communication appears to be similar in children with TSC without ASD and in children with TSC/ASD, thus suggesting a common developmental deficit in all children with TSC. ${ }^{32}$ In any case, the more severe are the deficits, the more likely is a diagnosis of ASD. ${ }^{32}$

\section{Potential Models of Early Interventions}

A presymptomatic diagnosis of TSC, when possible long before the age of 24 months, could facilitate not only the prospective investigation of developmental trajectories and early markers of ASD but also the evaluation of the efficacy of early interventions.

\section{Parent-Mediated Interventions}

Preclinical and clinical studies in high-risk populations of both idiopathic and syndromic forms of ASD revealed that enhanced environmental stimulations and an early intervention, designed to promote early social engagement and reciprocity, might remit or reduce the expression of symptoms. ${ }^{33-35}$ Therefore, the initiation of an intensive, targeted, and evidence-based behavioral intervention before the age of 2 years-when brain plasticity is at its maximum-is of crucial importance. Treatment strategies should be individualized and set up already before the onset of ASD symptoms, as it is happening with other high-risk populations, with the aim of improving social communication and other developmental domains. ${ }^{32,36}$

Several prospective studies on siblings of children with ASD have also demonstrated that improvements in parentinfant synchrony could lead to improvements in child dyadic communication and reduction in ASD symptoms. ${ }^{35,37}$ Parent-infant relationship-focused intervention could be a good model of treatment also for TSC children, as it seems to be able to elicit better functional communication and relationship and to stimulate social brain circuitry.

In the context of the above-mentioned EPISTOP project, our research group developed a preliminary protocol of parentimplemented interventions for infants with TSC. According to this protocol, all children undergo periodic neuropsychological assessments of autistic symptoms with ADOS-2-Autism Diagnostic Observation Schedule-2nd edition-and of developmental level with Bayley scale, to early identify deficits in specific developmental domains and to design individualized treatment strategies mediated by parents; the aim is to reduce autistic symptoms and to foster more typical developmental patterns. Our protocol has been designed on the basis of published studies on infants at high risk for idiopathic autism, such as ASD siblings, ${ }^{36}$ with the aim of increasing parental sensitivity and responsivity to infant cues. These are particularly important for enrichment in communication and the social development. - Table 1 summarizes the most important markers of an increased risk of ASD and the related parentimplementation strategies.

Table 1 Parent-mediated treatment approach for target symptoms in TSC infants at high risk for autism

\begin{tabular}{|l|l|}
\hline Unusual target behaviors & Actions to be taken by parents \\
\hline Unusual visual fixations on objects & $\begin{array}{l}\text { Follow infant interest to an object } \\
\text { Facilitate attention shifting from object to parent } \\
\text { Develop a turn-taking social game } \\
\text { Develop a parallel play and sharing of emotion regarding the object }\end{array}$ \\
\hline $\begin{array}{l}\text { Unusual repetitive patterns } \\
\text { of object exploration }\end{array}$ & $\begin{array}{l}\text { Encouraging exible and varied actions with the object } \\
\text { Show another pattern of movement } \\
\text { Follow infant interest while developing age-appropriate sensory-motor schemes for } \\
\text { object play } \\
\text { Shape motor movements into communicative gestures (e.g., turn repetitive hand } \\
\text { movements in a social clap) }\end{array}$ \\
\hline $\begin{array}{l}\text { Lack of intentional } \\
\text { communicative acts }\end{array}$ & $\begin{array}{l}\text { Offer and follow the child into preferred activities and dyadic and triadic joint activities } \\
\text { Elicit communicative gestures for asking again the activities } \\
\text { Press for integrated communicative behaviors (e.g., wait for the baby look at parent and } \\
\text { use the voice before giving the object) }\end{array}$ \\
\hline $\begin{array}{l}\text { Lack of age-appropriate } \\
\text { phonemic development }\end{array}$ & $\begin{array}{l}\text { Increase frequency of child vocalizations and shape specific consonant and vowel with } \\
\text { musical toys or games } \\
\text { Use imitation of infant babbling } \\
\text { Promote dyadic turn talking interactions }\end{array}$ \\
\hline $\begin{array}{l}\text { Decreasing gaze, social interest, } \\
\text { and engagement }\end{array}$ & $\begin{array}{l}\text { Position self and child for maximal face to face orientation and provide object and social } \\
\text { games that follow infant preferences } \\
\text { Maximize gaze and increase infant pleasure and engagement in social interaction }\end{array}$ \\
\hline
\end{tabular}

Note: In the first column, we report the main unusual behaviors in communicative and social interaction areas that could be indicative of high risk of autism and should be implemented by parents; in the second column principal strategies that could be taken by parents in daily activities are summarized.

Source: Adapted from Rogers et al 2014. ${ }^{36}$ 


\section{Targeted Pharmacological Treatment with mTOR Inhibitors}

The involvement of mTOR in determining the behavioral phenotypes associated with TSC led to the hypothesis that mTOR inhibitors, a class of drug selectively inhibiting mTOR complex and including rapamycin and everolimus, could also have a benefit on ASD symptoms. ${ }^{38}$ Preclinical studies demonstrated that the administration of rapamycin to a mouse model of early-onset epilepsy and autism was not only able to reduce seizure susceptibility but also to attenuate autisticlike behaviors. ${ }^{39,40}$ Besides animal models, there is also some initial clinical evidence of benefits of mTOR inhibitors on ASD symptoms in some patients. ${ }^{39}$ There is a critical need to evaluate the efficacy of mTOR inhibition in infants and young children with TSC, so to understand if ASD symptoms might benefit from this targeted treatment. According to the clinicaltrials.gov database, there are at present three registered clinical trials studying mTOR inhibitors in TSC (Efficacy of RAD001/Everolimus in Autism and NeuroPsychological Deficits in Children with Tuberous Sclerosis Complex [RAPIT]: NCT01730209; Rapalogues for Autism Phenotype in TSC: A Feasibility Study [RAPT]: NCT01929642; Trial of RAD001 and Neurocognition in Tuberous Sclerosis Complex: NCT01289912).

\section{Future Perspectives}

Early identification of infants at high-risk for ASD, such as TSC infants, can allow designing individualized treatment strategies to address deficits in specific developmental domains associated with autism. Prospective neurodevelopmental assessments should be performed since the first months of age and should include standardized measures of autistic symptoms and developmental level. Neurodevelopmental assessment should be associated with a close neurological and EEG follow-up, which can help to study individual developmental trajectories and to identify possible correlations between clinical and biological markers of ASD that might forecast the long-term outcomes. For all these reasons, clinical trials on very early intervention in TSC infants at high risk for ASD are warranted.

Due to the greater plasticity of younger neural systems, early identification of autistic behaviors and early interventions with parent implementation in infants at high risk of ASD can enrich the developmental trajectories and ameliorate the disabling effects of ASD. There is also hope that mTOR inhibitors that target the developing brain might prevent or restore its functional and structural changes, reestablishing the impaired synaptic plasticity, and improving autism-like behavioral abnormalities.

TSC treatment could benefit from present and future research on the molecular mechanisms involved in the pathogenesis of the disease, improving both the course of epilepsy and of its most invalidating neuropsychiatric comorbidities, such as ASD.

\section{Acknowledgments}

A. Benvenuto, R. Moavero, and P. Curatolo participated in the EPISTOP study (www.epistop.eu) which is funded under the European Community's Seventh Framework Programme (FP7/2007- 2013) under Grant Agreement no. 602391.

\section{References}

1 van Slegtenhorst M, de Hoogt R, Hermans C, et al. Identification of the tuberous sclerosis gene TSC1 on chromosome 9q34. Science 1997;277(5327):805-808

2 European Chromosome 16 Tuberous Sclerosis Consortium. Identification and characterization of the tuberous sclerosis gene on chromosome 16. Cell 1993;75(7):1305-1315

3 Napolioni V, Moavero R, Curatolo P. Recent advances in neurobiology of Tuberous Sclerosis Complex. Brain Dev 2009;31(2): 104-113

4 Curatolo P, Moavero R, de Vries PJ. Neurological and neuropsychiatric aspects of tuberous sclerosis complex. Lancet Neurol 2015; 14(7):733-745

5 Chu-Shore CJ, Major P, Camposano S, Muzykewicz D, Thiele EA. The natural history of epilepsy in tuberous sclerosis complex. Epilepsia 2010;51(7):1236-1241

6 American Psychiatric Association. Report of DSM-5 Proposed Criteria for Autism Spectrum Disorder. Arlington, VA: American Psychiatric Publishing; 2013

7 Kim YS, Leventhal BL, Koh YJ, et al. Prevalence of autism spectrum disorders in a total population sample. Am J Psychiatry 2011; 168(9):904-912

8 Lai MC, Lombardo MV, Baron-Cohen S. Autism. Lancet 2014;383 (9920):896-910

9 Critchley M, Earl C. Tuberous sclerosis and allied conditions. Brain 1932;55:311-346

10 Wong V. Study of the relationship between tuberous sclerosis complex and autistic disorder. J Child Neurol 2006;21(3):199-204

11 Numis AL, Major P, Montenegro MA, Muzykewicz DA, Pulsifer MB, Thiele EA. Identification of risk factors for autism spectrum disorders in tuberous sclerosis complex. Neurology 2011 76(11):981-987

12 Goorden SM, van Woerden GM, van der Weerd L, Cheadle JP, Elgersma Y. Cognitive deficits in Tsc $1+1-$ mice in the absence of cerebral lesions and seizures. Ann Neurol 2007;62(6):648-655

13 Ehninger D, de Vries PJ, Silva AJ. From mTOR to cognition: molecular and cellular mechanisms of cognitive impairments in tuberous sclerosis. J Intellect Disabil Res 2009;53(10):838-851

14 Curatolo P, Napolioni V, Moavero R. Autism spectrum disorders in tuberous sclerosis: pathogenetic pathways and implications for treatment. J Child Neurol 2010;25(7):873-880

15 Zeng LH, Ouyang Y, Gazit V, et al. Abnormal glutamate homeostasis and impaired synaptic plasticity and learning in a mouse model of tuberous sclerosis complex. Neurobiol Dis 2007;28(2):184-196

16 Bourgeron T. A synaptic trek to autism. Curr Opin Neurobiol 2009; 19(2):231-234

17 Jansen FE, Vincken KL, Algra A, et al. Cognitive impairment in tuberous sclerosis complex is a multifactorial condition. Neurology 2008;70(12):916-923

18 Bombardieri R, Pinci M, Moavero R, Cerminara C, Curatolo P. Early control of seizures improves long-term outcome in children with tuberous sclerosis complex. Eur J Paediatr Neurol 2010;14(2): 146-149

19 Cusmai R, Moavero R, Bombardieri R, Vigevano F, Curatolo P. Longterm neurological outcome in children with early-onset epilepsy associated with tuberous sclerosis. Epilepsy Behav 2011;22(4): 735-739

20 Curatolo P, Aronica E, Jansen A, et al. Early onset epileptic encephalopathy or genetically determined encephalopathy with early onset epilepsy? Lessons learned from TSC. Eur J Paediatr Neurol 2016;20(2):203-211 
21 Zhang B, McDaniel SS, Rensing NR, Wong M. Vigabatrin inhibits seizures and mTOR pathway activation in a mouse model of tuberous sclerosis complex. PLoS One 2013;8(2):e57445. Doi: 10.1371/journal.pone.0057445

22 Jóźwiak S, Nabbout R, Curatolo P; participants of the TSC Consensus Meeting for SEGA and Epilepsy Management.Management of subependymal giant cell astrocytoma (SEGA) associated with tuberous sclerosis complex (TSC): clinical recommendations. Eur J Paediatr Neurol 2013;17(4):348-352

$23 \mathrm{Wu}$ JY, Peters JM, Goyal M, et al. Clinical Electroencephalographic Biomarker for Impending Epilepsy in Asymptomatic Tuberous Sclerosis Complex Infants. Pediatr Neurol 2016; 54:29-34

24 Jóźwiak S, Kotulska K, Domańska-Pakieła D, et al. Antiepileptic treatment before the onset of seizures reduces epilepsy severity and risk of mental retardation in infants with tuberous sclerosis complex. Eur J Paediatr Neurol 2011;15(5): 424-431

25 Zeng LH, Rensing NR, Zhang B, Gutmann DH, Gambello MJ, Wong M. Tsc2 gene inactivation causes a more severe epilepsy phenotype than Tsc1 inactivation in a mouse model of tuberous sclerosis complex. Hum Mol Genet 2011;20(3):445-454

26 Cusmai R, Chiron C, Curatolo P, Dulac O, Tran-Dinh S. Topographic comparative study of magnetic resonance imaging and electroencephalography in 34 children with tuberous sclerosis. Epilepsia 1990;31(6):747-755

27 Bolton PF, Park RJ, Higgins JN, Griffiths PD, Pickles A. Neuroepileptic determinants of autism spectrum disorders in tuberous sclerosis complex. Brain 2002;125(Pt 6):1247-1255

28 Dabora SL, Jozwiak S, Franz DN, et al. Mutational analysis in a cohort of 224 tuberous sclerosis patients indicates increased severity of TSC2, compared with TSC1, disease in multiple organs. Am J Hum Genet 2001;68(1):64-80

29 Moavero R, Napolitano A, Cusmai R, et al. White matter disruption is associated with persistent seizures in tuberous sclerosis complex. Epilepsy Behav 2016;60:63-67

30 Peters JM, Sahin M, Vogel-Farley VK, et al. Loss of white matter microstructural integrity is associated with adverse neurological outcome in tuberous sclerosis complex. Acad Radiol 2012;19(1): $17-25$

31 Spurling Jeste S, Wu JY, Senturk D, et al. Early developmental trajectories associated with ASD in infants with tuberous sclerosis complex. Neurology 2014;83(2):160-168

32 Jeste SS, Varcin KJ, Hellemann GS, et al. Symptom profiles of autism spectrum disorder in tuberous sclerosis complex. Neurology 2016;87(8):766-772

33 Woo CC, Donnelly JH, Steinberg-Epstein R, Leon M. Environmental enrichment as a therapy for autism: A clinical trial replication and extension. Behav Neurosci 2015;129(4):412-422

34 Favre MR, La Mendola D, Meystre J, et al. Predictable enriched environment prevents development of hyper-emotionality in the VPA rat model of autism. Front Neurosci 2015;9:127. Doi: 10.3389/fnins.2015.00127

35 Koegel L, Singh A, Koegel R, Hollingsworth J, Bradshaw J. Assessing and improving early social engagements in infants. J Posit Behav Interv 2014;16(2):69-80

36 Rogers SJ, Vismara L, Wagner AL, McCormick C, Young G, Ozonoff S. Autism treatment in the first year of life: a pilot study of infant start, a parent-implemented intervention for symptomatic infants. J Autism Dev Disord 2014;44(12):2981-2995

37 Green J, Charman T, McConachie H, et al; PACT Consortium. Parent-mediated communication-focused treatment in children with autism (PACT): a randomised controlled trial. Lancet 2010; 375(9732):2152-2160

38 Ehninger D, Han S, Shilyansky C, et al. Reversal of learning deficits in a Tsc $2+1-$ mouse model of tuberous sclerosis. Nat Med 2008; 14(8):843-848

39 Talos DM, Sun H, Zhou X, et al. The interaction between early life epilepsy and autistic-like behavioral consequences: a role for the mammalian target of rapamycin (mTOR) pathway. PLoS One 2012;7(5):e35885. Doi: 10.1371/journal.pone.0035885

40 Kilincaslan A, Kok BE, Tekturk P, Yalcinkaya C, Ozkara C, Yapici Z. Beneficial Effects of Everolimus on Autism and Attention-Deficit/ Hyperactivity Disorder Symptoms in a Group of Patients with Tuberous Sclerosis Complex. J Child Adolesc Psychopharmacol 2016. Doi: 10.1089/cap.2016.0100 